\title{
Graph Search for Cloud Network Management
}

\author{
Misbah Uddin, Rolf Stadler \\ ACCESS Linnaeus Center \\ KTH Royal Institute of Technology \\ Email: \{ahmmud,stadler\}@kth.se
}

\author{
Masanori Miyazawa, Michiaki Hayashi \\ KDDI R\&D Laboratories Inc. \\ 2-1-15 Ohara, Fujimino City, Saitama Prefecture, Japan \\ Email: \{ma-miyazawa,mc-hayashi\}@kddilabs.jp
}

\begin{abstract}
A large part of operational and configuration information in networks and clouds has graph structure, e.g., virtual network topologies, IP flows, communication links of distributed cloud applications. The final objective of this work is to develop a scalable management system that allows realtime management applications, such as network analytics and anomaly detection applications, to search for graph-structured operational information. The paper contains a step towards this objective. The key challenge is to devise an efficient and scalable search process on data that is volatile and distributed across the network infrastructure. Approaches that are currently pursued for distributed graph databases are not applicable in this context. This paper presents design options and possible solutions for engineering a scalable graph search system that supports management applications. It includes a simple information model based on property graphs and a search query language based on keyword search. The architecture of the system centers around a distributed search plane that performs query processing using a network of search nodes. Finally, the paper outlines the design of a search node, which contains a local database that maintains graph partitions.
\end{abstract}

Keywords-graph search, network search, distributed query processing, distributed management, in-network management.

\section{INTRODUCTION}

A large part of operational and configuration information of networked systems, including computing and telecom clouds, has graph structure. Management applications read and modify such information, for instance, network topologies, paths of IP flows, broadcast trees, and communication patterns between virtual machines in a cloud application. For many management tasks, it is convenient to think in terms of searching and extracting graph information from the underlying infrastructure: for example, a quality assurance application which requires operational statistics from devices along a path of an IP flow, or a performance management application which traces a specific cloud service and retrieves location and identity of physical and logical infrastructure entities that run this application, together with resource usage and utilization data.

In order to make available graph-structured configuration and operational information to management applications, a traditional approach consists of maintaining this data in databases of the management infrastructure and refreshing the data using monitoring protocols and interfaces, such as SNMP or NetFlow. The drawback of such an approach is twofold. First, it does not scale to large-scale cloud infrastructures with hundreds of thousands of elements, which become increasingly common. Second, the volatility of the operational information requires an often infeasible amount of computational and networking resources to support management applications that can react rapidly on current state information. To prevent this drawback, one option is maintaining a distributed graphstructured database inside the network infrastructure. In this case, network information is accessed through a graph query language. We realized such an approach in the RDStore prototype [8]. RDStore is based on a commercial graph database system, which supports the Gremlin graph query language [1].

The issue with graph database systems available today, whether commercial products or research prototypes, is that they do not scale. The fundamental problem is captured by the CAP theorem, which states that no system can simultaneously posses the properties of consistency, availability and partition tolerance [5]. In fact, many research efforts in distributed graph databases are directed towards relaxing consistency to some degree, in order to make the system scalable, e.g., in terms of response times to queries.

For many management applications, transactional consistency in terms of ACID properties [6] is not required. The information base we are interested in includes monitoring data, device states and statistics, etc., which relate to graphstructured objects. For the management applications we envision, the freshness of this data is more important than the consistency of information across devices. In fact, we consider a very weak notion of consistency in our design. Our model includes operational information in the form of graphs, which are partitioned across a set of socalled search nodes. The partitions of a graph are updated locally through sensors and measurement probes and are not synchronized across nodes. Queries from management applications are global and are processed against local databases without synchronization across these databases. Consider the path of an IP flow whose graph representation is partitioned across a set of search nodes in network elements. On each node, sensors update the node statistics of this IP flow. A global query requesting the maximum load of any node along this path will be processed in a parallel fashion using subqueries against each local database.

In this paper, we present the design of a scalable graph search system for management of cloud networks. 


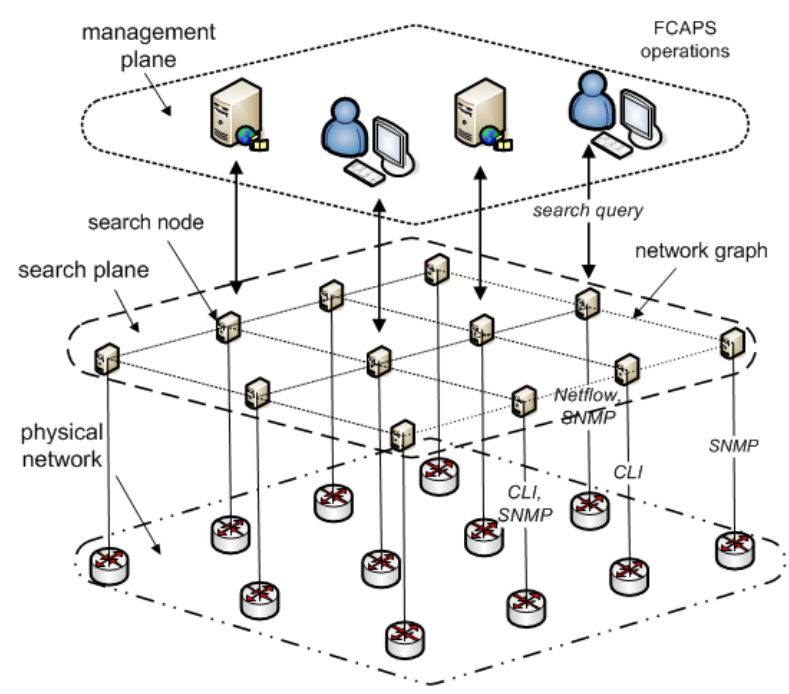

Fig. 1. An architecture for a graph search system [15]

Our design includes a simple information model that is based on property graphs [10] to represent operational and configuration data. Further, it includes a search query language that is based on keyword search and extended with boolean, projection and aggregation operators. The key component of the architecture is a distributed search plane that performs query processing using a network of search nodes. The query processing is performed using an echo protocol, which allows for scalable operation. Finally, we outline the design of a search node, which contains a local database that maintains graph partitions.

This work can be seen as an extension of our earlier work in designing a system for network search. The key extension is that network information is be structured in graph form, which impacts the query language, query processing, and node design [13] [14]. It allows to search for and search in large graph objects that represents important operational and configuration information in networked systems.

\section{Architecture of a Graph Search System}

Figure 1 shows the architecture for a graph search system that we developed for in-network processing of management information and applied to network search [15]. Its key element is the search plane, which conceptualizes the graph search functionality. This plane contains a network of search nodes, which have processing and storage capacities. A search node can communicate with a set of neighbors, which are identified through links of the network graph. The design of this plane supports searching in a distributed and parallel fashion. A search node can be realized in various ways: it can be part of the management infrastructure outside the managed system, it can be run as a standalone network appliance, or it can be integrated into a network element using a variety of technologies.

The bottom plane in Figure 1 represents the physical network that is subject to search. Each network element is associated with a search node, which maintains (or has access to) configuration and operational data from that network element. This data is modeled as a set of objects, whose structure is described in Section 3. Note that the figure shows the simplest form of association between a network element and a search node; it is possible that a search node maintains data from several physical devices, or, alternatively, a device updates data on several search nodes. The top of Figure 1 shows the management plane, which includes the systems and servers running processes for network supervision and management.

There are three important interfaces in this architecture. The first is the search interface, which supports the query language discussed in Section 4. We envision that every search node is an access point for search queries. The second interface defines the interaction between a search node and a network element, which can be realized through polling or can be push based. This interface is technologydependent and possibly proprietary. The third interface is the peer interface between the neighbouring search nodes. It enables nodes to interact for distributed processing of search queries.

Each search node runs a process that communicates with the associated network element(s) from which it retrieves network data. A database function dynamically maps that data into the information model for graph search and updates the local search database.

Search functions, invoked from the management plane through query invocation, are executed as distributed algorithms on the graph of search nodes. During the execution of a query on a search node, the local search database is accessed, the matching of the local query against stored indices is performed, and the local search result is possibly aggregated with results from other nodes.

\section{An INFORMATION MOdel FOR Graph SEARCH}

We model configuration and operational data in a networked system using the model of property graphs, which has been widely adopted for graph databases [10]. A property graph is composed of labeled vertices and edges. An edge is directed and, thus, has a start and end vertex. Both vertices and edges have properties, which are usually expressed as attribute-value pairs. Property graphs are often used to represent graph structures, for instance, the semantic web, social networks, etc.

We model the search space for a networked system as a set of property graphs. Each of these graphs represents an entity that is of interest to network management tasks. Examples of such entities are IP flows, virtual networks of various types, and virtual topologies. Many of them consist of only a single vertex, such as virtual machines, servers, and firewalls. We model a vertex as a set of attribute-value pairs that capture configuration parameters and operational states and statistics of an entity. Similarly, we model an edge as a set of attribute-value pairs that express, for instance, a communication channel, a link, or a hosting relationship. Each property graph, as well as each vertex and each edge, has a unique name and has a type. In our model, we encode names as uniform resource identifiers [3]. 


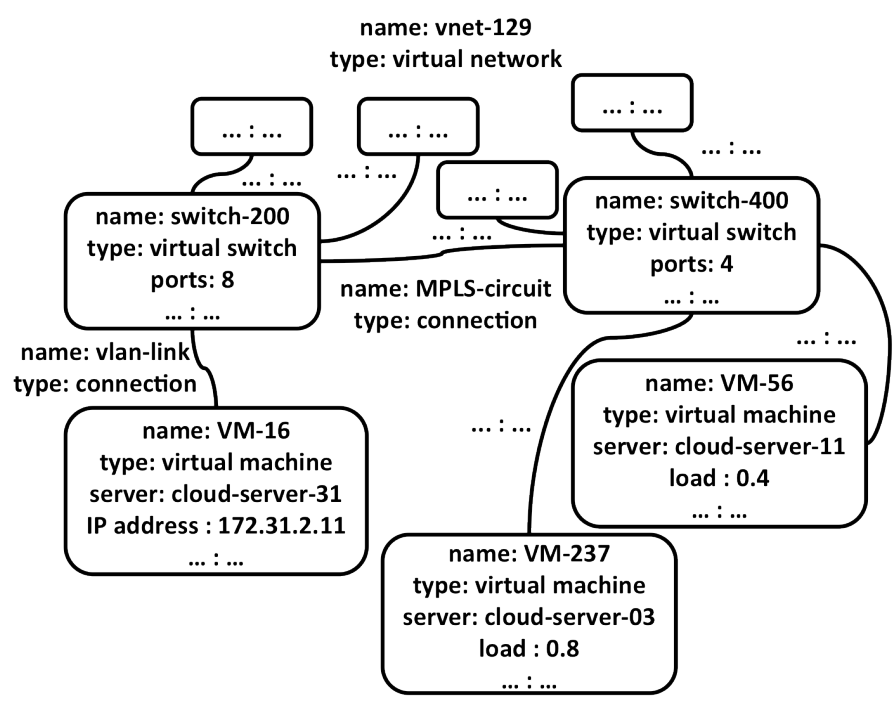

Fig. 2. A property graph representing a virtual network consisting of virtual switches, virtual machines, and virtual connections. Such graphs form the search space of a cloud network.

Figure 2 shows an example of a property graph in the search space of a cloud network. The name of the graph is 'vnet-129' and its type 'virtual network'. The figure includes some of the vertices and edges of this graph. Two such vertices are 'Switch-200' and 'VM-16'. They represent a virtual switch and a virtual machine, respectiviely. The edge 'vlan-link' represents a communication link between the virtual switch and the virtual machine.

An alternative to property graphs, popular in the web and database communities, is the resource description framework (RDF) [2]. Graphs are encoded in RDF as a set of triples of the form (subject, predicate, object). Each triple represents a labeled edge between a start node and an edge node. Compared to the property graph model, RDF has a simpler structure, but requires a larger graph to express a network entity. We chose property graphs for our information model, because the abstraction level that RDF graphs provide is too low for expressing network entities.

\section{A Query LANGUAGE FOR GRAPH SEARCH}

In this section, we describe the syntax and semantics of a query language that can be used by management processes to query the search space for graphs. The syntax of the query language is given in BNF notation [4]:

$$
\begin{aligned}
\text { Basic: } & q \rightarrow t|q \wedge q| q \vee q \\
& t \rightarrow a|v| a \text { op } v \\
& o p \rightarrow=|<|> \\
\text { Projection: } & p \rightarrow q \mid \pi q \\
\text { Aggregation: } & r \rightarrow p \mid \alpha p
\end{aligned}
$$

Our query language can be seen as an extension of a language for keyword search. The simplest query contains an attribute name, for example, 'IP address', or a value, for example, '172.31.2.11'. We allow also attribute-value pairs as queries, for instance, 'IP address $=172.31 .2 .11$ ' (rule
(2)). Whenever one of these queries matches an attributevalue pair of a vertex or an edge in a graph, the entire graph is included in the query result.

Queries can be constructed using terms (like attribute name, attribute value, attribute-value pair) and boolean operators (rule (1)). Two examples of such queries are '172.31.2.11 $\vee$ 172.31.3.23' and 'virtual network $\wedge$ IP address $=172.31 .2 .11$ '. While the former query returns the union of the subquery results, the latter returns the intersection of the subquery results. The graph in Figure 2 , for example, would match both of the above queries.

Our query language includes two specific operators for projection and aggregation (rules (4), (5)). We explain their syntax and semantics through examples. The projection query $\pi_{\text {load }}^{\text {vertex type }=V M}($ vnet -129$)$ retrieves all graphs that match 'vnet-129', selects from these graphs the vertices of type VM, and retrieves from those vertices the values of the attributes named 'load'. In other words, the query would retrieve the loads of the virtual machines for the specified virtual network (if there is a single graph matching the term 'vnet-129'). Similarly, the aggregation query $\alpha_{\text {max } \text { load }}^{\text {vertex }}$ (vnet-129) would retrieve all graphs that match 'vnet-129', would select from these graphs the vertices of type VM, and would compute the maximum value of the attributes named 'load' across the vertices. In other words, the operation would return the maximum load of the virtual machines in a specified virtual network (if there is a single graph matching the term 'vnet-129').

The above given semantics of the query language uses so-called exact matching, which is common for databasetype applications. For management applications that explores the search space, for instance, approximate matching is more appropriate. In our earlier work, we have developed a scheme for approximate matching and ranking in the context of network search [14]. This scheme is also applicable to graph search.

\section{Distributed Query Processing}

The search space for graph search is distributed among the local databases contained in the search nodes (see Figure 1). We anticipate that most graphs in the search space will be partitioned among search nodes, whereby each search node contains the subgraph whose information relates to its associated device(s). Consider a graph representing an IP flow, which contains vertices and edges that capture its properties. The vertices are maintained on the search nodes associated with the routers along the flow's path. Each edge is maintained on the search node that contains the start vertex as well as the node that contains the end vertex. This means that the graph is edge-partitioned [9].

Queries expressed in our language are processed in the search plane in a distributed way. There are (at least) two approaches for distributed processing of search queries. The first centers around maintaining an index that maps search terms to search nodes. For each term, the index contains those nodes that maintain partitions of matching graphs. 
A scalable way of maintaining such an index is using a distributed key-value store, e.g., Cassandra [7]. When processing a search query, first, the index is used to identify the search nodes that store relevant information. Then, graph information is retrieved from the local databases of those nodes. Lastly, the query result is computed using a distributed aggregation protocol. The challenge in this approach comes from the fact that most graphs contain operational data that is very volatile. As a consequence, maintaining such an index is very costly, if not infeasible. For this reason, this approach will not be pursued further here.

A second way of processing search queries is based on distributed search and involves all search nodes. In this context, an echo protocol can contribute to an efficient and scalable solution [12]. Echo, which implements an algorithm first described by Segall [11], is a tree-based protocol suitable for distributed polling. It executes on the network graph of the search plane (see Figure 1). It can be initiated on any search node. Once a node receives a search query from the management plane, echo disseminates the query to all nodes, creating an ad-hoc spanning tree in the process with the initiating node as root. Each node, upon receiving the query, executes it against its local database. The results of all local database queries are incrementally aggregated along the spanning tree, and the result of the search query becomes available at the root node. The code for the local operations for query processing, together with the state of the operation, are captured in an object called the aggregator object of the echo protocol [12].

Processing a search query $q$ involves two consecutive executions of the echo protocol. The first execution returns the names of the graph objects that match the query, and the second execution computes the actual result of the search query. Consider a query $q$ with terms $t_{i}, i=1, \cdots, n$. Figure 3 shows the pseudocode of the aggregator for the first execution. The aggregator for the second execution is very similar and is omitted due to lack of space.

The state of the aggregator for the first execution is captured in the variable $s$, a vector whose components $s[i]$ contain names of graph objets that match the terms $t_{i}$. The procedure local() executes the terms $t_{i}$ against the local database $D$ and stores the names of the matched objects in $s$. The procedure aggregate() aggregates the local state $s$ with the state child-s of a child node. The procedure global() is perfumed on the root node and merges the set of names in the components of $s$, by performing set union or set intersection, depending on the type of boolean operator that combines the terms $t_{i}$ in query $q$.

The performance characteristics of distributed query processing are based on the performance properties of the echo protocol [12].

\section{A Graph Search System for a Cloud NETWORK}

A cloud network typically includes data centers that host cloud services and an IP core network that provides con-
1: aggregator object process-query( )

var: $s$ : vector of names;

procedure local( )

$$
\begin{aligned}
& s:=\text { null; } \\
& \text { for each } t_{i} \in q \text { do: } \\
& \quad s[i]:=\pi_{\text {graph-name }}\left(t_{i}(D)\right) ;
\end{aligned}
$$

procedure aggregate(child-s: vector of names)

for each $t_{i} \in q$ do: $s[i]:=s[i] \cup$ child $-s[i]$

procedure global( )

$r:=$ merge $s[i], i=1, . ., n$;

return $(r)$;

Fig. 3. Aggregator for the first execution of the echo protocol, for a search query $q$ with terms $t_{i}, i=1, \cdots, n$.

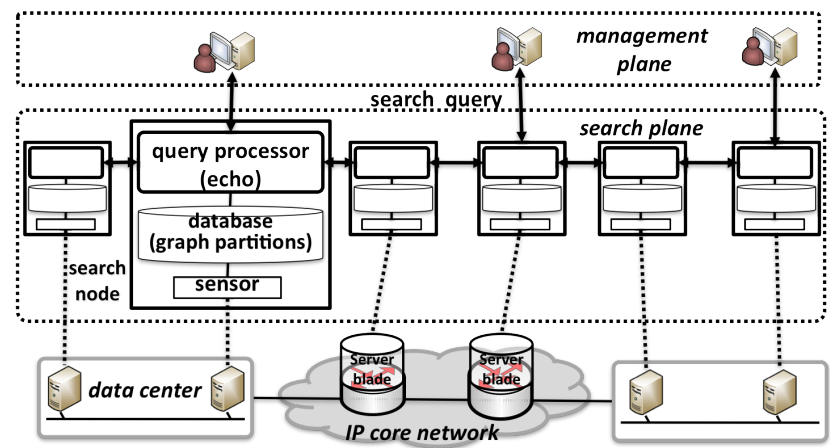

Fig. 4. A graph search system for a cloud network. Search nodes run on servers in data centers and on routers in the networking infrastructure.

nectivity between them. Figure 4 outlines the architecture of a graph search system for a cloud network.

We envision that a search node will execute on servers in data centers, as well as on network elements in the IP core network (for instance, on server blades). We have developed a database and sensing technology for graph management, which runs on a server blade of a large IP router [8] and which is suitable as a database component for a search node. We believe that search nodes in data centers do not require dedicated servers, as they can run efficiently using only a few percentage of the server resources [14].

\section{DiscusSION}

In this paper, we presented design options and solutions towards engineering a graph search system for network management. The key challenge in engineering such a system is devising an efficient search process that (a) supports expressive search queries like those expressed in our language, (b) provides fast response times to support real-time management applications, and (c) scales to the size of modern cloud infrastructures, which have in excess of a hundred thousands of nodes. In future work, we plan to complement the design outlined in this paper and build a prototype system for graph search based on our current implementations for in-network search [14] and in-network data stores [8]. 


\section{REFERENCES}

[1] Gremlin. https://github.com/tinkerpop/gremlin/wiki, last accessed: August 2013.

[2] Resource description framework (rdf). http://www.w3.org/RDF/, last accessed: July 2013.

[3] URIs, URLs, and URNs: clarifications and recommentations 1.0. http://www.w3.org/TR/uri-clarification/, last accessed: July 2013.

[4] Lars Marius Garshol. Bnf and ebnf: What are they and how do they work? http://www.garshol.priv.no/download/text/bnf.html, last accessed: July 2013.

[5] Seth Gilbert and Nancy Lynch. Brewer's conjecture and the feasibility of consistent, available, partition-tolerant web services. SIGACT News, 33(2):51-59, June 2002.

[6] Jim Gray. The transaction concept: virtues and limitations (invited paper). In Proceedings of the seventh international conference on Very Large Data Bases - Volume 7, VLDB '81, pages 144-154. VLDB Endowment, 1981.

[7] Eben Hewitt. Cassandra: The Definitive Guide. O'Reilly Media, 2010.

[8] Masanori Miyazawa and Michiaki Hayashi. Rdstore: In-network resource datastore with distributed processing of resource graph. In Integrated Network Management (IM 2013), 2013 IFIP/IEEE International Symposium on, pages 708-711, 2013.

[9] Francois Pellegrini. Graph Partitioning, chapter 4, pages 81-114. ISTE Wiley, 2011.

[10] I Robinson, J. Webber, and E. Eifrem. Graph Databases. O’Reilly Media, Inc., 2013.

[11] Adrian Segall. Distributed network protocols. IEEE Transactions on Information Theory, 29(1):23-34, 1983.

[12] R. Stadler. Protocols for distributed management. Technical Report 2012:028, KTH, Communication Networks, 2012. QC 20120604.

[13] M. Uddin, R. Stadler, and A. Clemm. A query language for network search. In IEEE/IETF international symposium on integrated network management, Ghent, Belgium, 27-31 May 2013.

[14] M. Uddin, R. Stadler, and A. Clemm. Scalable matching and ranking for network search. In 9th International conference on network and service management. Zurich, Switzerland, 14-18 October 2013

[15] M. Uddin, R. Stadler, and A. Clemm. Management by network search. In IFIP/IEEE Network Operations and Management Symposium (NOMS 2012), Maui, Hawaii, April 16 - 20, 2012. 